\title{
La competencia "Diseño y Proyecto" coordinada en la Mecánica de Fluidos de Ingeniería Química en los campus de la UPV: resultado de un Proyecto de Innovación y Mejora Educativa
}

Modesto Pérez-Sánchez ; Juan Manzano Juarez'; Rosa M. Llacer-Iglesias ${ }^{\mathrm{a}}$; Jorge García-Serra García ${ }^{\text {, }}$ P. Amparo López-Jiménez ${ }^{a}$,"

a Departamento de Ingeniería Hidráulica y Medio Ambiente. Universitat Politècnica de València. mopesan1@upv.es; rollaig@hma.upv.es; palopez@upv.es;

${ }^{b}$ ITA. Departamento de Ingeniería Hidráulica y Medio Ambiente. Universitat Politècnica de València. jgarcias@ita.upv.es.

c Departamento de Ingeniería Rural y Agroalimentaria. Universitat Politècnica de València. juamanju@agf.upv.es.

(*) autor de correspondencia

\begin{abstract}
The student's learning should be connected to correct coordination within the curriculum in which is taught. This coordination increases its complexity when the same Bachelor's Degree is taught in two different Campus of the Universitat Politècnica de València (Alcoy and Vera's Campus). This communication shows the results of the developed coordination in the matter Fluid Mechanics, which is taught in Chemical Bachelor's Degree. The obtained results are getting and documenting for the development of innovation project and improvement of the education (PIME), which is currently developing. The work shows the advantages of the transversal and vertical coordination, which affect on the student positively. Likewise, the coordination establishes the development of the programming of the Matter in both Campus, showing the good practice sheet, which enables to develop the proposed project. This sheet contains a rubric with new indicators, which were adapted to knowledge area of hydraulic engineering. This sample of coordination ca be extrapolated to other matters, sensing the student, homogeneity in the criteria and methodologies of active learning in the UPV generic student outcomes.
\end{abstract}

Keywords: design and Project; cross coordination, vertical coordination, rubric, UPV generic student outcomes

\section{Resumen}

El aprendizaje del alumno debería estar sujeto a una buena coordinación dentro del plan de estudios donde se imparte. Esta coordinación se hace más compleja cuando una misma titulación se imparte en dos Campus diferentes de la Universitat Politècnica de València (Alcoy y Vera). La comunicación 
La competencia "Diseño y Proyecto" coordinada en la Mecánica de Fluidos de Ingeniería Química en los campus de la UPV: resultado de un Proyecto de Innovación y Mejora Educativa

presentada muestra el resultado de coordinación de la asignatura Mecánica de Fluidos del Grado de Ingeniería Química. Este resultado se ha conseguido y documentado dentro del Proyecto de Innovación y Mejora en la Educación que actualmente se está desarrollando. El trabajo muestra las ventajas de la coordinación horizontal y vertical, la cual repercute de forma positiva al estudiante. Así mismo, establece el desarrollo de la programación de la asignatura en ambos Campus, mostrando la hoja de buenas prácticas que permite desarrollar el proyecto propuesto. Esta hoja contiene una rúbrica con nuevos indicadores que se adaptan de una forma más adecuada al área de la ingeniería hidráulica. Esta muestra de coordinación puede ser extrapolada a otras asignaturas, percibiendo el alumno, una homogeneidad en los criterios y metodologías de trabajo de aprendizaje activo en las competencias transversales.

Palabras clave: diseño y proyecto, coordinación horizontal, coordinación vertical, rúbrica, planificación

\section{Introducción}

El trabajo de la competencia transversal 'Diseño y Proyecto' en términos generales, pretende que el alumno aprenda integrando conocimientos y habilidades de diferentes ámbitos disciplinares aplicados al campo de trabajo concreto en que se aplique, desarrollando habilidades intelectuales de alto nivel, promoviendo el aprendizaje y trabajo autónomo, el trabajo en equipo y la autoevaluación, aplicando a su vez criterios de responsabilidad ética y profesional.

De esta forma, esta estrategia puede implementarse en muchos ámbitos de los actuales planes de estudios, donde la adquisición de competencias es también muy valorada por los docentes y empleadores. En la presente contribución se va a describir particularmente su propuesta de aplicación en el campo de las materias relacionadas con la Mecánica de Fluidos en las diferentes titulaciones de Ingeniería Química impartidas en los campus de la UPV.

Diferentes autores han trabajado esta competencia en diferentes niveles de forma individual (Part-Escriva,2015; Gonzalvez-Zafrilla et al., 2015; Lajara-Camilleri et al., 2015; Rullán et al, 2010). Sin embargo, son menos las experiencias que analizan el trabajo a un mismo nivel de dominio de la competencia cuando se trata de la misma titulación en diferentes Campus.

Particularmente, las materias relacionadas con la mecánica de fluidos son especialmente importantes en las titulaciones de Ingeniería Química. El comportamiento de los fluidos como medios continuos cuando se encuentran en reposo (estática) o cuando se encuentran en movimiento (cinemática y dinámica de fluidos) son aspectos fundamentales que afectan a prácticamente todas las operaciones básicas de un ingeniero químico. Así pues, la aplicación de la competencia se propone para estudiantes de grado, de segundo curso, donde se ubica la materia que se considera, por lo que el docente se encuentra frente a una aplicación de la competencia en la fase final del primer nivel de dominio. El desarrollo de

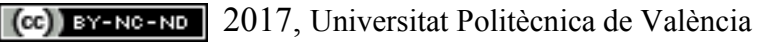


la asignatura y aprendizaje del alumno se basa en un contenido que en algunas fases tiene una importante componente teórica (Streter, 1963; White, 2008) una vez el alumno ya tiene fundamentadas las bases de la física y la matemática necesarias

Este nivel incluye acciones por parte del alumno, tales como: diseñar una instalación a nivel de anteproyecto; indicadores, justificar razonadamente la necesidad del proyecto, establecer los objetivos claros del proyecto planteado, proponer y programar en el tiempo las acciones para la consecución de los objetivos e identificar posibles riesgos inherentes al proyecto.

Asimismo, el caso de la UPV es particular, en tanto que la misma titulación se imparte en dos campus diferentes, separados espacialmente en dos provincias de la Comunidad Valenciana, Campus de Vera (Valencia) y Campus de Alcoy (Alicante), con dos Estructuras Responsable de Título (ERT) diferentes, de manera que la coordinación en la titulación se hace fundamental para asegurar que los contenidos que se imparten son similares y que los alumnos pueden intercambiarse en todo momento. Esta coordinación ha sido mejorada y documentada, como consecuencia del desarrollo del Proyecto de Innovación y Mejora en la Educación “B07. Desarrollo de Metodologías de Evaluación y Diseño de Rúbricas de la 'CT-05 Diseño y Proyecto' aplicado al campo de la Ingeniería Hidráulica” durante el curso 2016/2017.

La competencia "Diseño y Proyecto" se relaciona con una metodología de enseñanza y aprendizaje en la que los estudiantes desarrollan un trabajo completo en un tiempo determinado, con el objetivo fundamental de abordar una tarea mediante la planificación, diseño y realización de una serie de actividades (De Miguel, 2005). Se implementan así los conocimientos prácticos adquiridos, donde el proceso autónomo de aplicación a la solución de problemas tiene gran importancia, para dar una solución que puede ser abierta y diferente para cada alumno o grupo de ellos.

Para que los alumnos desarrollen un proyecto se necesita integrar el aprendizaje que han adquirido previamente en varias áreas y materias (Barrio Perrotti et al, 2010). En este caso, los alumnos que se enfrenten a un problema de mecánica de fluidos deberán integrar sus conocimientos en aspectos de física como control de magnitudes, cambios de unidades, cálculos de fuerzas y momentos o determinación de velocidades y caudales; con otros de matemáticas como integración o análisis de sistemas de ecuaciones diferenciales. En este sentido, el alumno combina conocimientos que ha adquirido fragmentados en otras materias y les encuentra una aplicación práctica y real en el campo de la aplicación hidráulica, muy cercana a la experiencia profesional, que después desarrollará como egresado en ingeniería química.

\section{Objetivos}

El objetivo de la presente contribución es presentar la planificación desarrollada en la asignatura Mecánica de Fluidos del Grado de Ingeniería Química, la cual es coordinada de manera transversal en los Campus de Vera y Alcoy.

Esta planificación busca por un lado, tener una distribución temporal similar en cuanto a los contenidos teóricos desarrollados, puesto que ambas asignaturas tienen los mismos contenidos teóricos reflejados en la Guía Docente, al ser las dos, el mismo título de la 
La competencia “Diseño y Proyecto" coordinada en la Mecánica de Fluidos de Ingeniería Química en los campus de la UPV: resultado de un Proyecto de Innovación y Mejora Educativa

Universitat Politècnica de València. Por otro lado, la coordinación transversal entre centros, busca ir más allá, y trata de desarrollar un mismo proyecto (a nivel de domino I) en ambos Campus, donde el alumno, de forma no presencial, tutorizado por el docente y apoyado en las prácticas informáticas realizadas, pueda desarrollarlo. El proceso se ha iniciado este curso 2016/2017 dentro del PIME anteriormente citado, y tratará de complementarlo totalmente en el curso 2017/2018, momento en que el acto de evaluación del proyecto desarrollado será introducido dentro de la Guía Docente al igual que los contenidos teóricos.

En la fase actual, durante el curso 2016/2017, se ha formado los grupos de trabajo de los profesores responsables de asignatura y profesores de prácticas informáticas, y a partir de ellos, se ha desarrollado la metodología de trabajo a seguir para poder desarrollar el trabajo no presencial.

\section{Desarrollo de la innovación}

El desarrollo de la metodología de trabajo propuesta busca conseguir una coordinación de la transversalidad en caso de existir la misma asignatura en diferentes centros o titulaciones. Esta coordinación, definida en la sección 3.1, viene presentada para una asignatura en dos centros diferentes, pero puede ser extrapoladas a asignaturas diferentes que estén relacionadas, pero sean del mismo o de diferentes Departamentos, para evitar solapamientos que reduzcan el tiempo de aprendizaje del alumno y esto sea percibido por éste como una muestra de desorganización del título. La coordinación puede venir establecida por estrategias similares a la definida en la sección 3. 2..

\subsection{Coordinación horizontal entre centros con perspectivas a la coordinación vertical}

En el presente contexto, debemos entender la coordinación docente como el conjunto de medidas orientadas a facilitar y mejorar el proceso de adquisición de las competencias de una titulación por parte de sus estudiantes (Parra et al, 2011). En este sentido, la materia que se presenta es, de un lado, complementaria a los conocimientos previos o que se desarrollan en paralelo para los estudiantes de Ingeniería Química; y de otro lado, fundamental para materias que han de adquirir en cursos superiores tales como máquinas hidráulicas, análisis de dispersión de contaminantes u operaciones complejas en ingeniería química.

Así pues, la coordinación entre los docentes (y más entre los que pertenecen a campus de la misma universidad), es importante en varios aspectos. El alumno debe percibir que se han ordenado convenientemente los contenidos de las distintas asignaturas, se han homogeneizado los sistemas de evaluación, y se ha gestionado de forma coordinada la carga de trabajo entre asignaturas y a lo largo de cada curso. En este sentido, se hace particularmente útil, el desarrollo de tareas complejas organizadas en un proyecto que permitan integrar todos estos conocimientos con una correcta planificación del tiempo.

(c) EY-NC-ND 2017, Universitat Politècnica de València 
La coordinación es fundamental en los procesos de innovación, siempre teniendo en cuenta las competencias adquiridas y planificación del tiempo del alumno. Todos los procesos de innovación se verán profundamente comprometidos en su futuro inmediato si se dan en un entorno descoordinado. Por otra parte, la coordinación, debe ser facilitada desde los centros, venciendo el individualismo de algunos profesores y la libertad de cátedra mal entendida, problemas en ocasiones endémicos en nuestros sistemas docentes (García Martin, 2015).

La coordinación de los equipos docentes en asignaturas de un mismo curso (coordinación horizontal) afecta también a futuras materias curriculares del alumno en cursos subsiguientes. Esta debe ser entendida como coordinación vertical, referida a toda la titulación y relacionada con los objetivos generales de la misma, y su coherencia en cuanto a todos los aspectos de adquisición de competencias y contenidos (Agudo y Gonzalo, 2006). En este sentido, la presente contribución muestra el interés desde el grupo de profesorado promotor, para que la realización de diseño y anteproyecto de los alumnos en las materias relacionadas con la mecánica de fluidos, sirvan como base para similares metodologías de proyectos con alcance mayor implementadas en cursos superiores para los alumnos, o incluso en el desarrollo de su trabajo final de grado, finalmente necesario para la adquisición de la titulación.

\subsection{Diseño de la coordinación de la asignatura}

La coordinación de la asignatura Mecánica de Fluidos está focalizada en dos términos diferenciados. La coordinación presencial desarrollada en la teoría de aula, práctica de aula e informáticas y la coordinación no presencial que deriva de la propuesta del proyecto a desarrollar por los alumnos, la coordinación está resumida en la Figura 1. Aunque los contenidos teóricos vienen establecidos en la Guía Docente, así como el tiempo dedicado presencialmente a cada unidad didáctica, el desarrollo de coordinación establece como prioridades:

1) Definir unos resultados de aprendizaje comunes, tanto a nivel del proyecto planteado como de la asignatura.

2) Que el desarrollo sea paralelo en ambos centros, de forma que el alumno se encuentre en el mismo nivel de adquisición de aprendizaje durante el cuatrimestre, no existiendo diferencia de contenidos dependiendo el Campus donde se localice. 


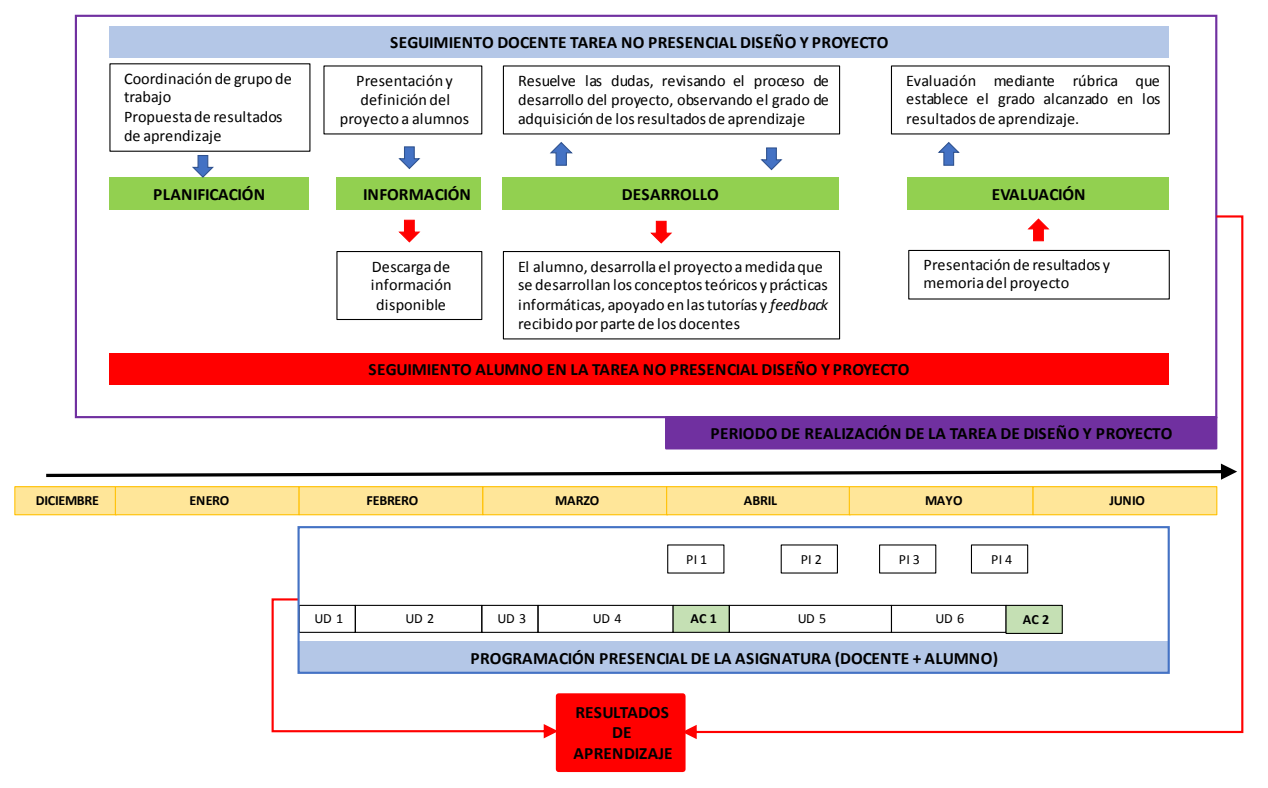

Fig. 1 Esquema temporal de desarrollo de acciones docentes y del alumnado

3) El proyecto está centrado en el Diseño de una red ramificada a flujo a presión, por lo que será abordado a partir del mes de Abril (desde la semana 10 hasta la semana 18). Iniciar la actividad al alumno en esta semana le permite:

- Recibir de manera síncrona los conceptos teóricos relacionados con el flujo a presión, relacionándolos con términos y aplicaciones desarrolladas en las unidades anteriores.

- De forma paralela a la teoría de aula y práctica de aula, el alumno realiza de forma presencial prácticas informáticas, donde el docente presenta un software con el cual poder analizar y comprobar redes de flujo a presión. Las prácticas informáticas están establecidas en cuatro sesiones de dos horas de duración en la EPSA y tres sesiones de $2.5 \mathrm{~h}$ en la ETSII.

- A medida que la docencia síncrona se desarrolla, el alumno comienza a desarrollar el proyecto. El desarrollo del proyecto se produce de forma no presencial. Es en esta etapa, donde el alumno comienza a mostrar el grado de alcanzado de aprendizaje, ya que debe aplicar al proyecto propuesto, los conceptos desarrollados presencialmente. Este aprendizaje no presencial, se sigue de forma tutorizada por los docentes a medida que se desarrolla el proyecto y el alumno lo solicita. Estas tutorías, sirven al alumno como 'feedback' para mejorar sus resultados de aprendizaje. Es en esta etapa, donde el alumno si lo desea puede complementar su aprendizaje mediante la utilización de recursos docentes asíncronos (p.e., Polimedias) que le ayuden a alcanzar con éxito sus resultados de aprendizaje (López-Jiménez y Pérez-Sánchez, 2016). No obstante, los alumnos deben ser, en la medida de lo posible, motivados para asistir a estas tutorías específicas, así como relacionar los conceptos teóricos y prácticos desarrollados en las clases 
presenciales, que son de aplicación directa al proyecto que deben desarrollar.

4) Permite medir el grado de adquisición de la competencia alcanzado por el alumno en su nivel I, atendiendo a los siguientes indicadores:

- Justificar razonadamente la necesidad del proyecto

- Establecer unos objetivos claros del proyecto

- Proponer las acciones para la consecución de los objetivos

- Desarrollar las acciones para la consecución de los objetivos

- Identificar posibles riesgos inherentes al proyecto

\section{Resultados}

El resultado en la presente comunicación no muestra los resultados alcanzados por los alumnos, sino el material generado por el docente para poder llevar a cabo esta coordinación transversal entre centros. Los resultados de dicha coordinación han sido documentados a través de:

- El desarrollo de la hoja de buenas prácticas de la asignatura, la cual permite en todo momento conocer a los docentes involucrados, las tareas a desarrollar (presenciales y no presenciales) así como la planificación temporal de las diferentes fases en la cual se encuentre el proyecto.

- Rubrica de evaluación que permita evaluar el proyecto desarrollado, definir el grado alcanzado en cada uno de los indicadores, permitiendo establecer una valoración descriptiva y no numérica.

\subsection{Hojas de buenas prácticas}

El desarrollo de la hoja de buenas prácticas ha permitido desarrollar la organización, de la asignatura, en base a la experiencia de los docentes implicados en años anteriores y a las reuniones de coordinación desarrolladas. La hoja de buenas prácticas ha sido implantada en el curso 2016/2017, lo que constituye un documento totalmente 'abierto', el cual irá ajustándose (fundamentalmente) los tiempos de dedicación de cada actor (docente y/o alumno) en próximos cursos.

Se resume a continuación, esta primera hoja de buenas prácticas coordinada entre centros, la cual conduce a la consecución de los resultados de aprendizaje a través del trabajo y adquisición de la competencia 'Diseño y Proyecto'.

- Título de Actividad: Dimensionado y análisis de una red ramificada de abastecimiento de agua a presión

- $\quad$ Asignatura: Mecánica de Fluidos; $2^{\circ}$ Curso; Cuatrimestre B; Troncal

- Número de Alumnos:

○ UPV-ETSII: 99 alumnos (1 grupo de TA y PA; 4 grupos de PI)

- UPV-EPSA: 47 alumnos (1 grupo de TA y PA; 2 grupos de PI)

\section{- Resultado de Aprendizaje del Proyecto planteado en la asignatura:}

○ Definir los objetivos por los cuales se desarrolla una red presurizada. 
La competencia "Diseño y Proyecto" coordinada en la Mecánica de Fluidos de Ingeniería Química en los campus de la UPV: resultado de un Proyecto de Innovación y Mejora Educativa

- Representar y describir todas las etapas del proceso de dimensionado de nuevas redes o ampliación de existentes, a partir de un diseño y trazado ya realizado.

o Dominar diferentes métodos de dimensionado (elección de diámetros nominales) de las tuberías de las redes de agua a presión ramificadas en sistemas de distribución urbanos o internos de una industria.

- Evaluar la idoneidad de los resultados obtenidos de forma numérica siguiendo los métodos propuestos, y proponer mejoras sobre el diseño inicial.

- Elegir el método de cálculo idóneo para cada caso, atendiendo a principios de calidad, garantía de suministro, económicas y sociales.

- Programación de la actividad desarrollada: a continuación, se adjunta la Tabla 1, donde se observa el tiempo dedicado a cada acción de acuerdo al desarrollo de la tarea y distribución temporal mostrada en la Figura 1, así como mostrando si es presencial o no presencial. Cada una de estas etapas está totalmente definida en la hoja de buenas prácticas, indicando las acciones de cada uno (docente o alumno) así como estableciendo las fechas 'deadline' de cada momento.

Tabla 1. Programación temporal de cada una de las acciones que intervienen en la actividad.

\begin{tabular}{|c|c|c|c|c|c|c|c|c|}
\hline \multirow{2}{*}{ FASES DE LA ACTIVIDAD } & \multicolumn{4}{|c|}{ DOCENTE } & \multicolumn{4}{|c|}{ ALUMNO } \\
\hline & PRESENCIAL & $t(\min )$ & $\begin{array}{c}\text { NO } \\
\text { PRESENCIAL }\end{array}$ & $t(\min )$ & PRESENCIAL & $t(\min )$ & $\begin{array}{c}\text { NO } \\
\text { PRESENCIAL }\end{array}$ & $t(\min )$ \\
\hline PLANIFICACIÓN & -- & -- & $\mathbf{X}$ & 300 & -- & -- & -- & -- \\
\hline \multicolumn{9}{|l|}{ INFORMACIÓN } \\
\hline $\begin{array}{l}\text { Elaboración de propuesta de } \\
\text { proyecto }\end{array}$ & -- & -- & $\mathbf{X}$ & 180 & -- & -- & -- & -- \\
\hline Presentación de proyecto & $\mathbf{X}$ & 30 & -- & -- & $\mathbf{X}$ & 30 & -- & -- \\
\hline $\begin{array}{l}\text { Descarga y recopilación de } \\
\text { información }\end{array}$ & -- & -- & -- & -- & -- & -- & $\mathbf{X}$ & 180 \\
\hline \multicolumn{9}{|l|}{ DESARROLLO } \\
\hline $\begin{array}{l}\text { TA, PA y PI relacionadas con el } \\
\text { proyecto }\end{array}$ & $\mathbf{X}$ & 1080 & -- & -- & $\mathbf{X}$ & 1080 & -- & -- \\
\hline Desarrollo del proyecto & -- & -- & -- & -- & & & $\mathbf{X}$ & 600 \\
\hline $\begin{array}{l}\text { Tutorías y análisis de la } \\
\text { evolución del aprendizaje }\end{array}$ & $\mathbf{X}$ & 720 & -- & -- & -- & -- & -- & -- \\
\hline \multicolumn{9}{|l|}{ EVALUACIÓN } \\
\hline $\begin{array}{l}\text { Presentación de resultados, } \\
\text { acorde a las instrucciones de la } \\
\text { actividad }\end{array}$ & -- & -- & -- & -- & -- & -- & $\mathbf{X}$ & 120 \\
\hline $\begin{array}{l}\text { Evaluación y determinación del } \\
\text { grado de aprendizaje alcanzado }\end{array}$ & -- & -- & $\mathbf{X}$ & 900 & -- & -- & -- & -- \\
\hline Feedback del proyecto & $\mathrm{X}$ & 100 & & & $\mathbf{X}$ & 100 & -- & -- \\
\hline
\end{tabular}

- Recursos: incluye la bibliografía recomendada, software EPANET, memorias de prácticas, documentos adjuntos en la tarea de PoliformaT (enunciado del proyecto, datos de partida para cada alumno, notas y aclaraciones para la correcta resolución del proyecto, ejemplo de plantilla de resultados a entregar, guion de la memoria y rúbrica de evaluación).

- Evaluación: la evaluación viene establecida mediante rúbrica, la cual permite establecer el grado alcanzado en el aprendizaje en función de los indicadores propuestos. Esta rúbrica es definida en la sección siguiente.

\subsection{Rúbricas desarrolladas}

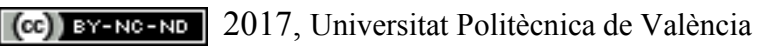


Los integrantes del PIME, en base a los indicadores establecidos por el Plan Estratégico de la UPV para Nivel de Dominio I, han desarrollado una rúbrica con nuevos indicadores, que permite evaluar los ítems englobados en el proyecto propuesto.

Tabla 2. Rubrica desarrollada para la evaluación de la competencia 'Diseño y Proyecto' Nivel de Dominio I

\begin{tabular}{|c|c|c|c|c|}
\hline \multirow{2}{*}{ INDICADORES } & \multicolumn{4}{|c|}{ DESCRIPTORES } \\
\hline & D. No alcanzado & C. En desarrollo & B. Bien /adecuado & A. Excelente/ejemplar \\
\hline $\begin{array}{c}\text { Define los } \\
\text { objetivos por los } \\
\text { cuales se } \\
\text { desarrolla una red } \\
\text { presurizada } \\
\end{array}$ & $\begin{array}{c}\text { No establece } \\
\text { objetivos o lo hace } \\
\text { incorrectamente }\end{array}$ & $\begin{array}{l}\text { Establece objetivos, pero } \\
\text { son ambiguos o imprecisos }\end{array}$ & $\begin{array}{c}\text { Establece objetivos de } \\
\text { forma suficiente }\end{array}$ & $\begin{array}{c}\text { Establece objetivos claros y } \\
\text { operativos }\end{array}$ \\
\hline $\begin{array}{l}\text { Introducción, } \\
\text { objetivos y } \\
\text { justificación }\end{array}$ & $\begin{array}{c}\text { No existe el primer } \\
\text { punto }\end{array}$ & $\begin{array}{c}\text { Establece objetivos, pero } \\
\text { son ambiguos o imprecisos }\end{array}$ & $\begin{array}{l}\text { Establece objetivos de } \\
\quad \text { forma suficiente }\end{array}$ & $\begin{array}{c}\text { Establece objetivos claros y } \\
\text { operativos }\end{array}$ \\
\hline $\begin{array}{c}\text { Representa y } \\
\text { describe todas las } \\
\text { etapas del proceso } \\
\text { de dimensionado } \\
\text { de nuevas redes }\end{array}$ & $\begin{array}{l}\text { No describe la } \\
\text { metodología de } \\
\text { cálculo } \\
\text { incorrectamente }\end{array}$ & $\begin{array}{l}\text { Describe la metodología, } \\
\text { pero no es correcta, } \\
\text { faltando alguno de los } \\
\text { procesos intermedios }\end{array}$ & $\begin{array}{c}\text { Describe la metodología } \\
\text { de cálculo } \\
\text { correctamente }\end{array}$ & $\begin{array}{l}\text { Describe la metodología de } \\
\text { cálculo correctamente y propone } \\
\text { alternativas o análisis de } \\
\text { sensibilidad al variar algún } \\
\text { parámetro sin desarrollarlo. }\end{array}$ \\
\hline $\begin{array}{l}\text { Para cada uno de } \\
\text { los puntos }\end{array}$ & $\begin{array}{l}\text { No existe ningún tipo } \\
\text { de introducción del } \\
\text { punto a desarrollar }\end{array}$ & $\begin{array}{c}\text { Hace una breve descripción } \\
\text { de lo contiene el punto }\end{array}$ & $\begin{array}{c}\text { Describe y justifica el } \\
\text { punto a desarrollar, pero } \\
\text { desde una perspectiva } \\
\text { académica }\end{array}$ & $\begin{array}{c}\text { Describe y justifica la necesidad del } \\
\text { punto a desarrollar desde un } \\
\text { punto de vista técnico }\end{array}$ \\
\hline $\begin{array}{l}\text { Domina diferentes } \\
\text { métodos de } \\
\text { dimensionado de } \\
\text { conducciones }\end{array}$ & $\begin{array}{l}\text { No es capaz de } \\
\text { desarrollar el método } \\
\text { de dimensionado }\end{array}$ & $\begin{array}{c}\text { Es capaz de iniciar el } \\
\text { proceso de dimensionado, } \\
\text { pero no consigue } \\
\text { concluirlo }\end{array}$ & $\begin{array}{l}\text { Consigue desarrollar el } \\
\text { método, extrae } \\
\text { resultados correctos, } \\
\text { pero no los justifica } \\
\text { completamente }\end{array}$ & $\begin{array}{l}\text { Consigue desarrollar el método, } \\
\text { extrae resultados correctos, pero } \\
\text { justificándolo completamente }\end{array}$ \\
\hline $\begin{array}{l}\text { Cálculo de los } \\
\text { caudales }\end{array}$ & $\begin{array}{c}\text { No aparece el punto } \\
\text { del cálculo de } \\
\text { caudales }\end{array}$ & $\begin{array}{l}\text { Se explica el método } \\
\text { seguido para calcular los } \\
\text { caudales y asignar las } \\
\text { demandas base a los } \\
\text { nudos, pero no se justifica }\end{array}$ & $\begin{array}{c}\text { Se explica y justifica el } \\
\text { método seguido para el } \\
\text { cálculo de los caudales }\end{array}$ & $\begin{array}{c}\text { Se explica y justifica el método } \\
\text { seguido para el cálculo de los } \\
\text { caudales. Se aporta una } \\
\text { valoración personal al método } \\
\text { seguido. }\end{array}$ \\
\hline $\begin{array}{l}\text { Dimensionado por } \\
\text { el método de la } \\
\text { velocidad }\end{array}$ & $\begin{array}{l}\text { No aparece el punto } \\
\text { del dimensionado }\end{array}$ & $\begin{array}{l}\text { Se explica el método } \\
\text { seguido para calcular los } \\
\text { diámetros, pero no se } \\
\text { justifica }\end{array}$ & $\begin{array}{l}\text { Se explica y justifica el } \\
\text { método seguido para el } \\
\text { cálculo de los diámetros }\end{array}$ & $\begin{array}{c}\text { Se explica y justifica el método } \\
\text { seguido para el cálculo de los } \\
\text { diámetros. Se aporta una } \\
\text { valoración personal al método } \\
\text { seguido. }\end{array}$ \\
\hline $\begin{array}{l}\text { Análisis de la red } \\
\text { con los resultados } \\
\text { obtenidos }\end{array}$ & $\begin{array}{c}\text { No aparece el punto } \\
\text { del análisis }\end{array}$ & $\begin{array}{c}\text { Se muestran los resultados } \\
\text { de las variables, pero no se } \\
\text { analizan }\end{array}$ & $\begin{array}{l}\text { Se analizan los } \\
\text { resultados y se } \\
\text { introducen posibles } \\
\text { mejoras }\end{array}$ & $\begin{array}{c}\text { Se analizan los resultados y se } \\
\text { realizan posibles mejoras, } \\
\text { comparando ambos estados de la } \\
\text { red }\end{array}$ \\
\hline $\begin{array}{l}\text { Dimensionado por } \\
\text { el método de la } \\
\text { pendiente } \\
\text { hidráulica }\end{array}$ & $\begin{array}{l}\text { No aparece el punto } \\
\text { del dimensionado }\end{array}$ & $\begin{array}{c}\text { Se explica el método } \\
\text { seguido para calcular los } \\
\text { diámetros, pero no se } \\
\text { justifica }\end{array}$ & $\begin{array}{l}\text { Se explica y justifica el } \\
\text { método seguido para el } \\
\text { cálculo de los diámetros }\end{array}$ & $\begin{array}{c}\text { Se explica y justifica el método } \\
\text { seguido para el cálculo de los } \\
\text { diámetros. Se aporta una } \\
\text { valoración personal al método } \\
\text { seguido. }\end{array}$ \\
\hline
\end{tabular}


La competencia "Diseño y Proyecto" coordinada en la Mecánica de Fluidos de Ingeniería Química en los campus de la UPV: resultado de un Proyecto de Innovación y Mejora Educativa

Tabla 3. Rubrica desarrollada para la evaluación de la competencia 'Diseño y Proyecto' Nivel de Dominio I [Continuación]

\begin{tabular}{|c|c|c|c|c|}
\hline \multirow{2}{*}{ INDICADORES } & \multicolumn{4}{|c|}{ DESCRIPTORES } \\
\hline & D. No alcanzado & C. En desarrollo & B. Bien /adecuado & A. Excelente/ejemplar \\
\hline $\begin{array}{l}\text { Evalúa la } \\
\text { idoneidad de los } \\
\text { resultados } \\
\text { obtenidos }\end{array}$ & No realiza el análisis & $\begin{array}{c}\text { Solo muestra resultados, } \\
\text { sin comentarlos }\end{array}$ & $\begin{array}{c}\text { Muestra resultados, } \\
\text { analizando los valores } \\
\text { obtenidos y causas a } \\
\text { las que se deben }\end{array}$ & $\begin{array}{l}\text { Muestra resultados, analizando } \\
\text { los valores obtenidos y causas a } \\
\text { las que se deben, proponiendo } \\
\text { mejoras e incluso abordándolas }\end{array}$ \\
\hline $\begin{array}{l}\text { Análisis de la red } \\
\text { con los resultados } \\
\text { obtenidos }\end{array}$ & $\begin{array}{c}\text { No aparece el punto } \\
\text { del análisis }\end{array}$ & $\begin{array}{l}\text { Se muestran los resultados } \\
\text { de las variables, pero no se } \\
\text { analizan }\end{array}$ & $\begin{array}{l}\text { Se analizan los } \\
\text { resultados }\end{array}$ & $\begin{array}{c}\text { Se analizan los resultados y se } \\
\text { realizan posibles mejoras, } \\
\text { comparando ambos estados de la } \\
\text { red }\end{array}$ \\
\hline $\begin{array}{c}\text { Elige el método de } \\
\text { cálculo idóneo } \\
\text { para cada caso, } \\
\text { atendiendo a } \\
\text { principios de } \\
\text { calidad, garantía } \\
\text { de suministro, } \\
\text { económicas y } \\
\text { sociales } \\
\end{array}$ & No lo realiza & $\begin{array}{c}\text { Selecciona método, pero } \\
\text { no justifica }\end{array}$ & $\begin{array}{l}\text { Selecciona método } \\
\text { atendiendo a análisis } \\
\text { comparativo }\end{array}$ & $\begin{array}{l}\text { Selecciona método atendiendo } \\
\text { a los resultados, y desarrolla } \\
\text { una comparación a través de } \\
\text { cálculos complementarios }\end{array}$ \\
\hline Conclusión & No existe conclusión & $\begin{array}{l}\text { La conclusión aparece } \\
\text { únicamente como último } \\
\text { punto del proyecto, sin } \\
\text { análisis ni crítica de los } \\
\text { resultados }\end{array}$ & $\begin{array}{c}\text { Se analizan los } \\
\text { diferentes resultados y } \\
\text { se comparan métodos }\end{array}$ & $\begin{array}{c}\text { Se analizan los resultados, } \\
\text { comparando ambos métodos e } \\
\text { identificando ventajas e } \\
\text { inconvenientes de cada uno de } \\
\text { ellos }\end{array}$ \\
\hline $\begin{array}{l}\text { Lenguaje, formato } \\
\text { y redacción del } \\
\text { proyecto }\end{array}$ & $\begin{array}{c}\text { La presentación es } \\
\text { deficiente, la } \\
\text { redacción y el } \\
\text { lenguaje utilizados no } \\
\text { se corresponden con } \\
\text { el nivel académico } \\
\text { esperado }\end{array}$ & $\begin{array}{l}\text { La presentación es } \\
\text { correcta, aunque } \\
\text { mejorable, el lenguaje y la } \\
\text { redacción no son técnicos }\end{array}$ & $\begin{array}{c}\text { La presentación, el } \\
\text { lenguaje y la redacción } \\
\text { son correctas. El } \\
\text { contenido excede o no se } \\
\text { ajusta a lo requerido }\end{array}$ & $\begin{array}{l}\text { La presentación, el lenguaje y la } \\
\text { redacción son correctas. El } \\
\text { contenido se ajusta } \\
\text { perfectamente a lo requerido para } \\
\text { explicar cada uno de los puntos }\end{array}$ \\
\hline
\end{tabular}

La evaluación se realiza atendiendo al contenido de la memoria y anexos presentados por el alumno, relacionándolos con el indicador correspondiente. Tal y como muestran las Tablas 2 y 3 , la rúbrica recoge la totalidad de los indicadores para evaluar la competencia, así como los apartados de los cuales se componen el proyecto y que los alumnos deben desarrollar a lo largo del cuatrimestre. La metodología, así como la rúbrica de evaluación planteada dotan al alumno de una base que le permite desarrollar la competencia en próximas asignaturas con una estructura similar relacionadas con el área de Mecánica de Fluidos o Ingeniería Hidráulica. Mantener una operatividad y sistemática de trabajo dota a la competencia de una transversalidad vertical que enriquece en un mayor grado la formación del estudiante.

\section{Conclusiones}

La presente comunicación ha mostrado la importancia de la existencia de coordinación transversal entre dos Campus de la UPV, con dos ERT diferentes, en una misma asignatura (Mecánica de Fluidos), en el Grado de Ingeniería Química. Esta coordinación se ha 
intensificado este curso 2016/2017 cuando la asignatura ha sido integrada en el PIME 'B07. Desarrollo de Metodologías de Evaluación y Diseño de Rúbricas de la 'CT-05 Diseño y Proyecto’ aplicado al campo de la Ingeniería Hidráulica”.

Sentar las bases de metodologías de trabajo y consecución de los objetivos en actividades de 'Diseño y Proyecto' en su nivel de Dominio I es fundamental para dotar al alumno de una estrategia de actuación que trasladada a otras asignaturas relacionadas con el área de estudio o similar le confieren a la competencia una coordinación vertical, en la cual el estudiante puede maximizar el alcance de los resultados de aprendizaje.

Esta transversalidad y verticalidad mostrada en este documento puede ser extendida a otras asignaturas que también trabajen dicha competencia y como actividad tengan propuestos proyectos en los cuales intervengan sistemas hidráulicos que necesiten de otros elementos complementarios (p.e., estructuras, instalaciones eléctricas para los grupos de bombeo).

Finalmente, una rúbrica ha sido desarrollada para Nivel de Dominio I, para llevar a cabo la evaluación del grado de adquisición de los resultados de aprendizaje del alumno en función de la actividad propuesta, la cual ha sido previamente definida en forma (estableciendo las funciones del docente y alumno, tanto presencial como no presencial) y tiempo (definiendo los tiempos de trabajo de cada fase).

\section{Referencias}

Agudo, C., Gonzalo, I. (2006). Coordinación docente: dónde estamos y a dónde querríamos llegar. Miscelánea Comillas. Vol. 64 (2006), núm. 124, pp. 63-82

Barrio Perotti, R., Blanco, E., Martínez de la Calle, J., Galdo Vega, M. (2010). RED. Docencia Universitaria en la Sociedad del Cono cimiento, número 2. http://www.um.es/ead/reddusc/2/

De Miguel, M. (2005). Metodologías de enseñanza para el desarrollo de competencias. Orientaciones para el profesorado universitario ante el Espacio Europeo de Educación Superior. Madrid: Alianza.

GARCIA MARTIN, A. (2015). Coordinación docente horizontal y vertical. Universidad Politécnica de Cartagena, España.

LÓPEZ-JIMÉNEZ, P.A., PÉREZ-SÁNCHEZ, M. "Los objetos de aprendizaje como conductores para favorecer el aprendizaje autónomo en el campo de la mecánica de fluidos”. II Congreso Nacional de Innovación Educativa y de Docencia en Red (Valencia, 7 y 8 de julio, 2016). Editorial Universitat Politècnica de València, 2016. Doi:http://dx.doi.org/10.4995/INRED2016.2016.4347

Parra Costa, C., Periago, P., García Baño, R., Maciá Sánchez, J.F., Peñalver Martínez, M.J., Martínez Segura, M.A., Múlas PÉrez, J., Doménech Asensi, G. (2011) Estrategias de coordinación horizontal y vertical en los planes de estudios adaptados al EEES. En: Congreso Internacional de Innovación Docente, Cartagena, 2011. Cartagena: Universidad Politécnica de Cartagena, 2011. p.1815-1824

Streeter, V.L., 1963. Mecánica de los Fluidos, Segunda. ed. Ediciones del Castillo SA, Madrid, Spain. 
La competencia "Diseño y Proyecto" coordinada en la Mecánica de Fluidos de Ingeniería Química en los campus de la UPV: resultado de un Proyecto de Innovación y Mejora Educativa

WHITE, F.M., 2008. Fluid Mechanics, Sixth edit. ed. McGrau-Hill. 